\title{
Tesla Turbine Experiment
}

\author{
Vishwam Shah $^{1}$, Shyam Dhokai ${ }^{2}$ \\ U.G. student, Department of Mechanical Engineering, Gujarat Technological University, India
}

\begin{abstract}
Nikola Tesla invented this bladeless turbine (patented 1913) originally, which uses boundary layer effect to run and not impingement of fluid upon the blades as in conventional. There are number of discs sequentially mounted on a shaft and the fluid is made to flow in a tangential direction with considerable pressure onto the discs with the help of an efficient nozzle, then follows a spiral path towards the centre and exits axially. The discs rotate due to the basic properties of the fluid - viscosity and adhesion, as momentum is transferred via this forces and kinetic energy of fluid is converted into the rotational energy of the shaft. Many researchers have studied this concept and given various improvements with proofs basically into two ways - one suggesting modifying the design of various components involved while others to change the parameters involved. Here a model is made of Tesla turbine and effort is made to understand on how to increase the efficiency by experimenting on a few parameters.
\end{abstract}

Keywords: Bladeless turbine, Boundary layer, plenum chamber

\section{Introduction}

In most of the mechanical systems, friction is generally avoided and effort is done to minimize it as less as possible. Friction reduces the efficiency of a system by a large factor and can result in several other disadvantages like wearing of the parts and heating components. Friction is not a fundamental force itself, it results from inter surface interaction moving relative to each other. Now, when a fluid is made to flow across a solid surface a thin layer of fluid in the immediate vicinity of the surface is in contact with it where the velocity of the flow varies from zero at the surface up to its original flow velocity. This thin layer of fluid is called boundary layer and the effect produced by it is called boundary layer effect. In aviation this boundary layer drag is a serious hindrance and reduces the efficiency up to $50 \%$. Nikola Tesla viewed this friction in a totally opposite manner and used it as a primary force to rotate the disks used in this bladeless turbine. Boundary layer is formed due to the basic properties of the fluid i.e. viscosity and adhesion. This produces shear force between the fluid and the surface which results in the torque acting on the disks of the turbine, hence rotating it.

High velocity fluid enters the turbine through inlet nozzle and hits the disks tangentially at its outer periphery making a boundary layer on either side of the disks. Now, the fluid spirals towards the centre due to pressure ratio of the device and exits axially after imparting all the energy of boundary drag to the disks of the turbine.

In this present work, a Tesla turbine is manufactured using simple materials. Experimental results are carried out using compressed air as a medium. The overall setup is flexible and adaptable to change like number of disks, spacing between them and angle of the nozzle.

\section{Parameters Affecting Performance}

\subsection{Power}

Tesla turbine is best suited for low power generation spectrum or where primary cost is critical or where fluid properties hinder the performance of conventional turbine.
Several experiments have been carried and all that concluded the same result of Tesla turbine outperforming the conventional ones at low power generation areas.

\subsection{Nozzle}

For the same pressure drop, diverging nozzle produces about one-third more horsepower than the straight nozzle.

It is an impulse turbine as all the expansion of steam occurs in the nozzle only rather than onto the rotors (as a result of which the exhaust holes are placed as nearer to the centre of the rotor).

The main drop in overall efficiency seen due to the less efficiency of the nozzle. This problem can be solved however by using plenum chamber which increases the stability and uniformity of the jet flow before entering into the nozzle.

\subsection{Flow Characteristics}

Presence of the turbulent flow conditions at the transition from laminar to turbulent till mid turbulent gives the best power output i.e. the Reynolds number approximately 450,000. (Medium- compressed air)

\subsection{Friction Factor}

Poiseuille number increases with the surface roughness. (By Gamarat)

This increase in the number is of substantial help here as it improves the performance of the rotor as the primary force of running the turbine is shear force.

The second benefit of increasing the roughness is the increase in the momentum transfer of the fluid to the discs thereby increasing the drag force and faster the tangential velocity drop.

\subsection{R.P.M}

Effectiveness of operating the turbine can be maximized by running at a lower rpm and more flow rate. This increases the momentum transfer of the fluid to the discs. 


\section{International Journal of Science and Research (IJSR) \\ ISSN (Online): 2319-7064}

Index Copernicus Value (2015): 78.96 | Impact Factor (2015): 6.391

\subsection{Interdisk Spacing}

This spacing can be more optimized but according to experimental results the minimum ratio of the rotor's radius to the inter-disc spacing should be 20 . According to Warren Rice, the highest efficiency is seen the turbine when the distance between the disks is equal to two times the boundary layer thickness.

\subsection{Number of Disks}

By increasing the number of disks, the surface area on which the fluid flows increases thereby increasing the torque acting and hence the efficiency.

\section{Experimentation}

\subsection{Shaft}

The shaft here is made up of aluminum as due to light weight and ease of rotation. Also, speeds are not going extreme so aluminum is the best suitable material here. Support is provided at the ends of the shaft via bearings. It is manufactured by simple machining on lathe.

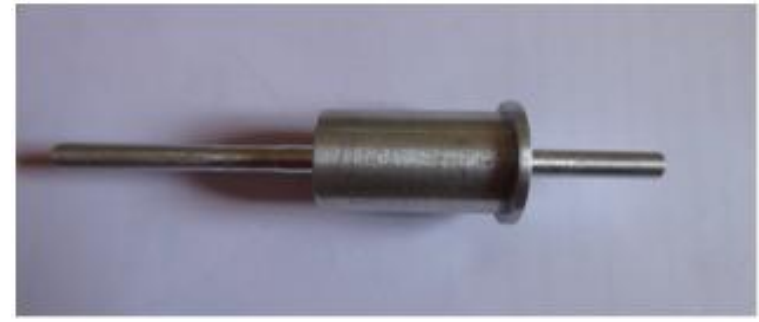

Figure 1: shaft

\subsection{Bearings}

Bearings are very critical component as they must be able to withstand considerable amount of heat and high speeds of rotation. For this experiment the bearings used were FAG ball bearings with $17 \mathrm{~mm}$ as inner diameter and $25 \mathrm{~mm}$ outer diameter as due to not such extreme speeds were expected.

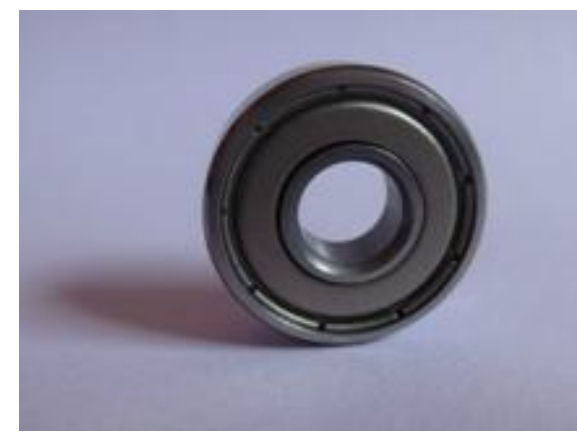

Figure 2: Bearing

\subsection{Rotor}

These are very important for system performance as they should be chosen considering various parameters depending on the conditions they are to be used. They depend on the factors such as inlet fluid to be used, the pressure and temperature of the system and also the moment of inertia required to be generated in the system. For instance, in high pressure turbines larger diameter rotors are required. Here low pressure air is used as fluid and conditions are also moderate. So, hard drive platters were used for this experiment for the ease of material sourcing and construction. Also, the size of the rotors is in direct comparison with the research done by Hoya and Guha (2009) and Guha and smiley (2009). They have a very smooth surface with extremely precise dimensions and were readily available with no additional cost. The material for rotors is aluminum. In larger disks with more rotational speeds are required, higher yield strength material should be preferred such as steel or carbon fiber.

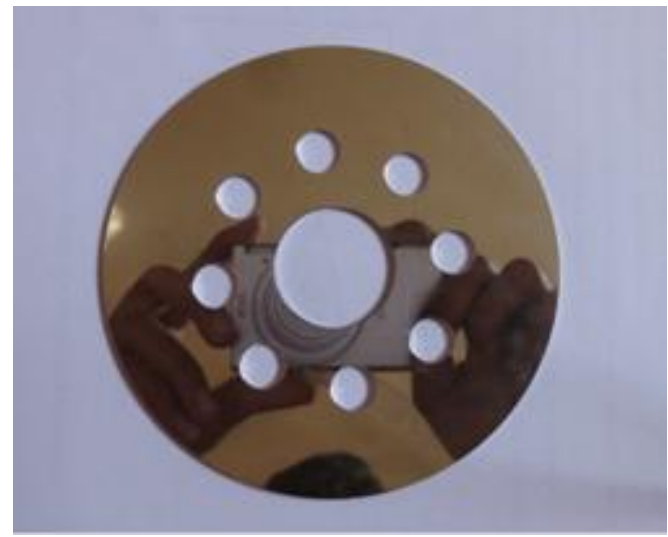

Figure 3: Rotor

\subsection{Spacers}

In the design, spacers are placed in between the disks and mounted on the shaft. The spacers are intended to be light weight and such as do not hinder the performance of rotation of disk.

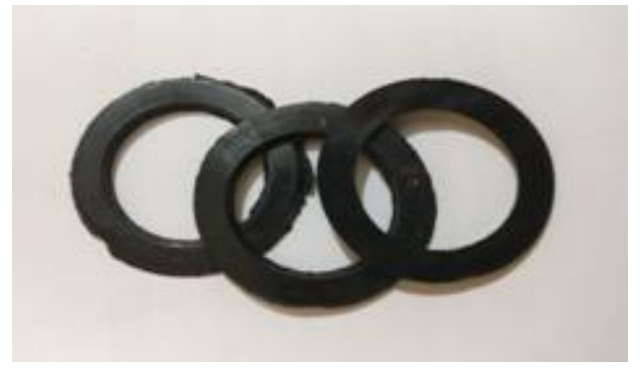

Figure 4: Spacers

\subsection{Disks Assembly}

Discs are mounted on shaft with help of collars which are shown below.

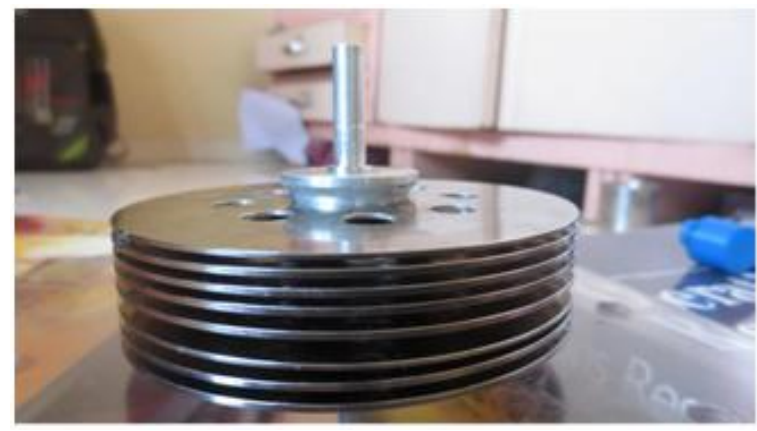

Figure 5: Disks assembly 


\section{International Journal of Science and Research (IJSR) \\ ISSN (Online): 2319-7064}

Index Copernicus Value (2015): 78.96 | Impact Factor (2015): 6.391

\subsection{Turbine Assembly}

Final assembly of the turbine was the easy combination of all the components fabricated and manufactured as shown above. The two plexi glasses were strongly fitted against the pvc pipe making an air tight casing suitable for our turbine with the help of full threaded bolts. Then a pipe for inlet was sealed into the inlet port using silicon sealant which served perfectly as a source of compressed air to enter tangentially on to the discs.

\section{Results}

After manufacturing the turbine, several test runs were performed and working conditions were observed to make out few conclusions. Below is given a table of all the experiments with readings of notable scientists with parameters and the readings that were taken during this experiment.

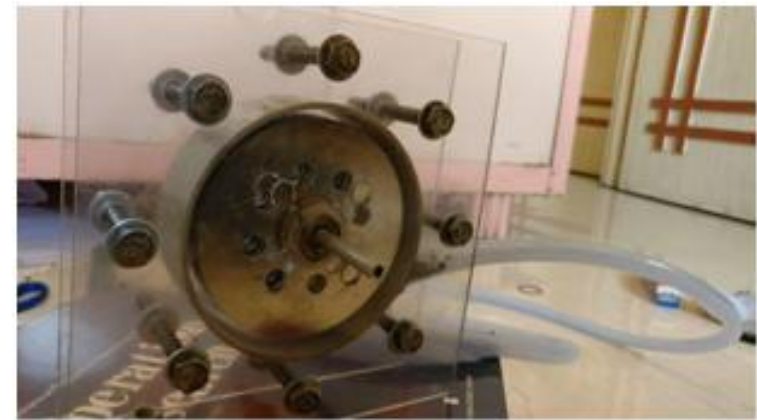

Figure 6: Turbine assembly

\begin{tabular}{|c|c|c|c|c|c|c|c|c|c|c|}
\hline Author & $\mathrm{r}_{0}$ & $\mathrm{r}_{i}$ & \begin{tabular}{|c|} 
central(c) \\
shaft(s) \\
exhaust
\end{tabular} & $\begin{array}{c}\# \\
\text { Discs }\end{array}$ & $\begin{array}{c}\text { Disc } \\
\text { Spacing }\end{array}$ & $\mid \begin{array}{c}\text { Steam(s) } \\
\text { Air }(\mathrm{a}) \\
\text { Water }(\mathrm{w})\end{array}$ & \begin{tabular}{|c|} 
Operating \\
Pressure
\end{tabular} & $\begin{array}{c}\max \\
\text { speed }\end{array}$ & $\begin{array}{c}\max \\
\text { torque }\end{array}$ & $\begin{array}{c}\text { max } \\
\text { power }\end{array}$ \\
\hline & $(\mathrm{mm})$ & $(\mathrm{mm})$ & & & $(\mathrm{mm})$ & & (bar) & (rpm) & $(\mathrm{Nm})$ & W \\
\hline Armstrong (1952) & 88.9 & 47.625 & $\mathrm{~s}$ & 10 & variable & $\mathrm{s}$ & 8.1 & 6000 & 0.1 & 530 \\
\hline Beans (1961) & 76.2 & 30.5 & $s$ & 6 & $12.7-.67$ & $a$ & 2.75 & 17000 & 0.9 & 5600 \\
\hline Rice (1963) & 88.9 & 33.5 & $\mathrm{C}$ & 9 & 1.6 & $\mathrm{a}$ & 9.6 & 11800 & $\mathrm{n} / \mathrm{a}$ & 1800 \\
\hline Leaman (1950) & 63 & 10 & $\mathrm{C}$ & 4 & 3.2 & $a$ & 5.8 & 9000 & 0.1 & 87 \\
\hline Lemma (2008) & 25 & 5.95 & $\mathrm{~s}$ & $6(?)$ & $\mathrm{n} / \mathrm{a}$ & $\mathrm{a}$ & 0.514 & 96000 & 0.04 & 220 \\
\hline Tesla (1913) & 228.5 & $\mathrm{n} / \mathrm{a}$ & $\mathrm{s}$ & 25 & $\mathrm{n} / \mathrm{a}$ & $\mathrm{s}$ & 8.6 & 9000 & $\mathrm{n} / \mathrm{a}$ & $150 \mathrm{k}$ \\
\hline Emran (2011) & 18.8 & 17 & $\mathrm{~s}$ & 4 & $\mathrm{n} / \mathrm{a}$ & $\mathrm{a}$ & 5.9 & 50000 & 0.345 & $\mathrm{n} / \mathrm{a}$ \\
\hline Peshlakai (2012) & 75 & 34.5 & $\mathrm{~s}$ & 12 & 1.3 & $\mathrm{a}$ & 6.14 & $\mathrm{n} / \mathrm{a}$ & $\mathrm{n} / \mathrm{a}$ & 12 \\
\hline Bloudicek (2007) & 100 & 17 & $\mathrm{C}$ & 13 & $\mathrm{n} / \mathrm{a}$ & $\mathrm{a}$ & 20.5 & $\mathrm{n} / \mathrm{a}$ & 0.7 & 58.3 \\
\hline Romanin (2012) & 36.5 & 18 & $\mathrm{~s}$ & 10 & 1.2 & $\mathrm{a}$ & 5.4 & 24170 & $\mathrm{n} / \mathrm{a}$ & $\mathrm{n} / \mathrm{a}$ \\
\hline Romanin (2012) & 5 & 1 & $\mathrm{C}$ & 4 & 0.5 & w & $\mathrm{n} / \mathrm{a}$ & $\mathrm{n} / \mathrm{a}$ & $\mathrm{n} / \mathrm{a}$ & 35 \\
\hline Hoya \& Guha (2009) & 46 & 12.5 & $\mathrm{C}$ & 8 & 0.2 & $a$ & 3.6 & 25000 & 0.7 & 140 \\
\hline
\end{tabular}

\begin{tabular}{|l|c|c|c|c|c|c|c|c|c|c|}
\hline This Experiment & 47.5 & 12.5 & c & 8 & 1 & a & 4 & 4500 & 0.9034 & 137.83 \\
\hline
\end{tabular}

\section{Conclusion}

Overall, the system performed better than expected. The initial inspection of the machined parts was disappointing, and there was concern that this would affect the ability for the turbine to operate reliably. With proper use of the analytical results, the rotor efficiency using laminar flow can be very high, even above $95 \%$.

However, in order to attain high rotor efficiency, the flow rate number must be made small which means high rotor efficiency is achieved at the expense of using a large number of disks and hence a physically larger rotor. For each value of flow rate number there is an optimum value of Reynolds number for maximum efficiency. With common fluids, the required disk spacing is dismally small causing [rotors using] laminar flow to tend to be large and heavy for a prescribed through flow rate.

\section{References}

[1] May, P., Tesla, N., \& York, N. E. W. (1913). » N. Tesla.

[2] W. Rice, "An Analytical and Experimental Investigation of Multiple Disk Turbines," Journal of Engineering for Power, vol. 87, pp. 29-36, 1965.

[3] Ho-yan, B. P. (2011). Tesla Turbine for Pico Hydro Applications, (4), 1-8.

[4] S. B. Guha A., "Experiment and analysis for an improved design of the inlet and nozzle in Tesla disc turbines," Journal Power and Energy, vol. 224, no. 2, pp. 261- 277, 2009.

[5] May, P., Tesla, N., \& York, N. E. W. (1913). » N. Tesla.

[6] W. Rice, "An Analytical and Experimental Investigation of Multiple Disk Turbines," Journal of Engineering for Power, vol. 87, pp. 29-36, 1965.

\section{Volume 6 Issue 7, July 2017 www.ijsr.net}




\section{International Journal of Science and Research (IJSR) \\ ISSN (Online): 2319-7064}

Index Copernicus Value (2015): 78.96 | Impact Factor (2015): 6.391

[7] Ho-yan, B. P. (2011). Tesla Turbine for Pico Hydro Applications, (4), 1-8.

[8] S. B. Guha A., "Experiment and analysis for an improved design of the inlet and nozzle in Tesla disc turbines," Journal Power and Energy, vol. 224, no. 2, pp. 261- 277, 2009.

[9] G. A. Hoya G. P, "The design of a test rig and study of the performance and efficiency of a Tesla disc turbine," Proceedings of the Institution of Mechanical Engineers, Part A: Journal of Power and Energy, vol. 223, pp. 451465, 2009.

[10] Taylor, J. B., Carrano, A. L., \& Kandlikar, S. G. (2006). Characterization of the effect of surface roughness and texture on fluid flow - past, present, and future 5 , 45 , 962-968. http://doi.org/10.1016/j.ijthermalsci.2006.01.004

[11] Gamrat, G., Favre-Marint, M., Le Person, S., Baviere, R., \& Ayela, F. (2008). An experimental study and modelling of roughness effects on laminar flow in microchannels. J. Fluid Mech, 594(October 2016), 399423. http://doi.org/10.1017/S0022112007009111

[12] Armstrong, J. H. (n.d.). an Investigation of the Performance of a Modified Tesla Turbine.

[13] Bott, R. (2014). Challenging the Versatility of the Tesla Turbine: Working Fluid Variations and Turbine Performance. Igarss 2014, (1), 1-5.

[14] Krishnan, V. G. (2015). Design and Fabrication of cmscale Tesla Turbines, (October). http://doi.org/10.1115/1.1413771

[15] Guha, A., \& Smiley, B. (2016). Experiment and analysis for an improved design, 224, 261-277. http://doi.org/10.1243/09576509JPE818

[16] Agrawal, S. K., Gardner, G., \& Pledgie, S. (2015). Design and Fabrication of an, 123(December 2001), 525-528. http://doi.org/10.1115/1.1413771

[17] Borate, H. P. (2012). An Effect of Spacing and Surface Finish on the Performance of Bladeless Turbine, (1), $1-7$. http://doi.org/10.1115/GTINDIA2012-9623 